\title{
Quantification of quantumness in neutrino oscillations
}

\author{
Fei Ming ${ }^{1}$, Xue-Ke Song ${ }^{1}$, Jiajie Ling ${ }^{2}$, Liu Ye ${ }^{1}$, Dong Wang ${ }^{1,3, a}$ (i) \\ ${ }^{1}$ School of Physics and Material Science, Anhui University, Hefei 230601, People's Republic of China \\ ${ }^{2}$ School of Physics, Sun Yat-Sen University, Guangzhou 510275, People's Republic of China \\ ${ }^{3}$ CAS Key Laboratory of Quantum Information, University of Science and Technology of China, Hefei 230026, People's Republic of China
}

Received: 2 January 2020 / Accepted: 13 March 2020 / Published online: 26 March 2020

(C) The Author(s) 2020

\begin{abstract}
Neutrino oscillation is an important physical phenomenon in elementary particle physics, and its nonclassical features can be revealed by the Leggett-Garg inequality. It shows that its quantum coherence can be sustained over astrophysical length scales. In this work, we investigate the measure of quantumness in experimentally observed neutrino oscillations via the nonlocal advantage of quantum coherence (NAQC), quantum steering, and Bell nonlocality. From various neutrino sources, ensembles of reactor and accelerator neutrinos are analyzed at distinct energies, such as Daya Bay $(0.5 \mathrm{~km}$ and $1.6 \mathrm{~km})$ and MINOS $(735 \mathrm{~km})$ collaborations. The NAQC of two-flavor neutrino oscillation is characterized experimentally compared to the theoretical prediction. It exhibits non-monotonously evolutive phenomenon with the increase of energy. Furthermore, it is found that the NAQC is a stronger quantum correlation than quantum steering and Bell nonlocality even in the order of $\mathrm{km}$. Hence, for an arbitrary bipartite neutrino-flavor state with achieving a NAQC, it must be also a steerable and Bell nonlocal state. The results might offer an insight into the neutrino oscillation for the further applications on quantum information processing.
\end{abstract}

\section{Introduction}

Dating from over half a century ago, the interesting phenomenon of the neutrino oscillation (NO) was discovered [1-5]. Afterwards, vast number of experimental evidences of neutrino flavor transitions have been achieved from various neutrino sources, i.e., solar neutrinos [6-8], atmospheric neutrinos $[9,10]$, reactor neutrinos $[11,12]$ and accelerator neutrinos [13-15]. According to the framework of threegeneration neutrino, neutrinos and antineutrinos simultaneously generate, and both of them can be detected in three flavors, viz., the electron-neutrino $v_{e}$, muon-neutrino $v_{\mu}$ and tau-neutrino $v_{\tau}$ (see the review on neutrino oscillations (NOs)

a e-mail: dwang@ahu.edu.cn (corresponding author) in Ref. [16]). Some efforts have been made to reveal interesting details of the physics of neutrinos, and measure and analyze the accurate oscillations parameters [17-19].

Typically, the flavor states are linear combinations of mass states [20,21]. Due to their nonzero mass and mixing, one flavor may oscillate to another flavor during propagation, which usually is called as NO. The NOs are basically threeflavor oscillations [22], which can be mapped into the states of neutrino, considering them as the three-mode systems, namely, the three-qubit systems [23]. From the normal order of the neutrino mass spectrum $\left(m_{1}<m_{2}<m_{3}\right)$, the mixing parameters of neutrino are $\Delta m_{21}^{2}=7.5 \times 10^{-5} \mathrm{eV}^{2}$, $\Delta m_{31}^{2}=2.457 \times 10^{-3} \mathrm{eV}^{2}, \Delta m_{32}^{2}=2.382 \times 10^{-3} \mathrm{eV}^{2}$, $\theta_{12}=33.48^{\circ}, \theta_{23}=42.3^{\circ}$, and $\theta_{13}=8.50^{\circ}[24,25]$. In some special cases, they can be also reduced to the effective two-flavor ones [26]. Similarly, they can be investigated via considering them as the two-mode systems, to that the two-qubit quantum systems.

The interactions of NOs are weak interactions, and the decoherence effects of NOs are very smaller than other particles. This stimulates the observations of NO in the domain of quantum information science. Whether it is possible to exploit the potential applications of NOs on quantum information processing? Before considering this, the quantumness of NOs should be adequately examined in advance. For this purpose, some works [27-30] have tested the quantumness of NOs by utilizing the Leggett-Garg inequality (LGI) treated as the temporal Bell inequality. It suggests that the classical limits can be violated with the LGI in experimentally observed NOs [31].

However, there exists some actual limitations for testing NOs by the LGI, initially taken advantage of certifying the theory of macroscopic realism with respect to macroscopic objects. If the system violates the LGI, it manifests that the system may not be a macroscopic reality or a noninvasive measurement cannot be implemented on it. Nevertheless, for the current experimental settings, these two conditions 
cannot be fully satisfied by the detection of elementary particles. Moreover, to quantify the magnitude of quantum coherence, the violation of the LGI is not always a good method, so that its violation can only be used as a criterion for quantumness. Of course, this is similar to the case that Bell inequality cannot be generally used to precisely quantify quantum entanglement [32-36].

More recently, based on coherence complementarity relations, the steerability of local coherence was presented by a game between a pair of participants, say Alice and Bob [37]. For a two-qubit state $\rho_{A B}$, based on local measurements on subsystem $A$ and classical communication between the bipartite, the average coherence of conditional state of subsystem $B$ could surpass the limit of the coherence as to the state of single-qubit measured in mutually-unbiased basis. A nonlocal advantage of quantum coherence (NAQC) can thus be attained by the conditional state of subsystem $B$. Afterwards, the NAQC for two-qubit states with high fidelity is experimentally demonstrated in an optics-based platform [38]. The information theoretic tools have many applications to solve the problems in NOs for the distinguishment the neutrinos nature between Dirac and Majorana fermions [39] and the mass-degeneracy problem [40]. Also, some progresses have been made on the correlation by virtue of flavor transition probabilities of NOs by quantum information theory, such as quantum discord [26], Svetlichny inequalities [25], concurrence $[41,42]$, quantum coherence [43], and quantum estimation [44].

In this paper, we focus on exploring the NAQC, quantum steering, and Bell nonlocality in experimentally observed NOs. It is found that the NAQC for neutrino-flavor states can be captured based on the $l_{1}$ norm of coherence. Comparing to the LGI, the NAQC, acting as a special quantum correlation, can effectively quantify genuine quantumness. We also investigate the relationship among NAQC, Bell nonlocality, and quantum steering in the dynamics of NO. The result shows that the nonlocal advantage of quantum coherence is a stronger quantum correlation compared with quantum steering and Bell nonlocality.

The paper is structured as follows. In Sect. 2, we briefly review the basic features of the neutrinos phenomenology and introduce the two-flavor mode entangled state analyzed via the tools of quantum information theory. In Sect. 3, we capture the NAQC, quantum steering, and Bell nonlocality in experimentally observed NOs. In Sect. 4, concise conclusions and discussions are given.

\section{Two-flavor NOs}

Three distinct neutrino flavors are components of the standard neutrinos model. Here we mainly pay attention to the energy and distance on oscillations in the method of two- state approximation [45]. In the relativistic limit, oscillations between the two-flavor states can be analyzed by the Bloch sphere formalism [46]. It geometrically denotes the space of the pure state of two-level quantum system.

The Hamiltonian for the neutrino propagation within the limit of two-flavor can be written as (setting $c=\hbar=1$ ) $[20,21,28,45]$

$$
\begin{aligned}
H= & \left(p+\frac{m_{1}^{2}+m_{2}^{2}}{4 p}+\frac{V_{C}}{2}+V_{N}\right) \mathbb{I} \\
& +\frac{1}{2}\left(\begin{array}{ll}
V_{C}-\omega \cos 2 \theta & \omega \sin 2 \theta \\
\omega \sin 2 \theta & \omega \cos 2 \theta-V_{C}
\end{array}\right) \\
= & r_{0} \mathbb{I}+\frac{\mathbf{r} \cdot \boldsymbol{\sigma}}{2},
\end{aligned}
$$

where $\theta$ stands for the neutrino vacuum mixing angle, $\sigma=$ $\left(\sigma_{x}, \sigma_{y}, \sigma_{z}\right)$ denotes a vector of two-dimensional Pauli matrices, $\mathbb{I}$ is a $2 \times 2$ identity matrix, and $p \simeq E$ represents the relativistic neutrino momentum-energy, $m_{1}$ and $m_{2}$ are the distinct mass states, respectively, and $\omega=\left(m_{2}^{2}-m_{1}^{2}\right) / 2 p$ denotes the oscillation frequency. $V_{C(N)}=\sqrt{2} G_{F} n_{e(n)}$ corresponds to the charged (neutral) current potential because of coherent forward scattering of neutrinos with electrons (neutrons) in matter, with $G_{F}$ representing the Fermi coupling constant. The term $r_{0} \mathbb{I}$ is proportional to $\mathbb{I}$ and has the identical influences on all flavor states, and hence has no contribution to flavor oscillations. For the neutrinos with the given energy $E_{k}$, the unitary operator $U$ governing the time evolution of the flavor states related to $H_{o s c} \equiv \mathbf{r} \cdot \sigma / 2$ reads

$$
\begin{aligned}
U\left(\omega_{k} ; t_{i} ; t_{j}\right) & \equiv U\left(\psi_{k ; i j}\right)=\exp \left(-i \int_{t_{j}}^{t_{i}} H_{o s c}\left(\omega_{k}\right) d t\right) \\
& =\cos \left(\psi_{k ; i j}\right)-i \sin \left(\psi_{k ; i j}\right)\left(\hat{r}\left(\omega_{k}\right) \cdot \sigma\right),
\end{aligned}
$$

where $\omega_{k}$ corresponds to the oscillation frequency for the energy $E_{k}$ and $\psi_{k ; i j} \equiv\left|\mathbf{r}\left(\omega_{k}\right)\right|\left(t_{j}-t_{i}\right) / 2$ denotes the accumulated phase in the propagating period from $t_{i}$ to $t_{j}$ with the energy $E_{k}$. In the limit that the matter effects are still negligible, $\psi_{k ; i j}$ can be given by

$\psi_{k ; i j} \simeq \frac{\omega_{k}}{2}\left(t_{j}-t_{i}\right)=\frac{1}{4 E_{k}}\left(m_{2}^{2}-m_{1}^{2}\right)\left(t_{j}-t_{i}\right)$.

The time evolution of neutrino is only related to the accumulated phase $\psi_{k ; i j}$, not the individual times $t_{i}$ and $t_{j}$. The time-dependent evolution of flavor neutrino states can be computed by

$\left|v_{\alpha}(t)\right\rangle=U\left(\omega_{k} ; t_{i} ; t_{j}\right)\left|v_{\alpha}\right\rangle=a_{\alpha \alpha}(t)\left|v_{\alpha}\right\rangle+a_{\alpha \beta}(t)\left|v_{\beta}\right\rangle$,

with $\alpha, \beta=e, \mu$ for electron- and muon-flavor neutrino states. $\left|v_{\alpha}\right\rangle$ is the flavor state at the initial time $t=0$. The 
probabilities (the survival probability $v_{\alpha} \rightarrow v_{\alpha}$ and the oscillation probability $v_{\alpha} \rightarrow v_{\beta}$ ) on the neutrino in states $\left|v_{\alpha}\right\rangle$ and $\left|v_{\beta}\right\rangle$ are $P_{\alpha \alpha}=\left|a_{\alpha \alpha}(t)\right|^{2}$ and $P_{\alpha \beta}=\left|a_{\alpha \beta}(t)\right|^{2}$, respectively, and the probabilities satisfy $P_{\alpha \alpha}+P_{\alpha \beta}=1$. The occupation number states of neutrinos can be introduced by the following correspondence $[23,26]$

$\left|v_{\alpha}\right\rangle \equiv|1\rangle_{\alpha} \otimes|0\rangle_{\beta} \equiv|10\rangle$,

$\left|v_{\beta}\right\rangle \equiv|0\rangle_{\alpha} \otimes|1\rangle_{\beta} \equiv|01\rangle$.

Therefore, the time evolution of the flavor eigenstate can be treated as the two-qubit state

$\left|v_{\alpha}(t)\right\rangle=a_{\alpha \alpha}(t)|10\rangle+a_{\alpha \beta}(t)|01\rangle$.

\section{NAQC, Bell nonlocality, and quantum steering in experimentally observed NOs}

Now, let us briefly review the NAQC based on the $l_{1}$ norm of coherence. First, we introduce the so-called incoherent state and the incoherent operation. The theoretical architecture of the NAQC was set up that the incoherent state is one which is diagonal in the given reference basis, while the operation is called as the incoherent operation that can map the incoherent state into the incoherent state [47]. The coherence measure is defined by a distance to the closest incoherent state. The $l_{1}$ norm of coherence in the basis of eigenvectors of Pauli spin observables $\sigma_{i}(i=x, y, z)$ is reformulated as

$C_{l_{1}}^{\sigma_{i}}(\rho)=\sum_{R \neq S}\langle R|\rho| S\rangle$,

where $\{|R\rangle,|S\rangle\}$ are the eigenvectors of $\sigma_{i}$. In Ref. [37], the complementarity relation of coherence is given under mutually unbiased bases by

$\sum_{i=x, y, z} C_{l_{1}}^{\sigma_{i}}(\rho) \leq C_{\max }$

where $C_{\max }$ is the state-independent upper bound with the value $C_{\max }=\sqrt{6} \approx 2.45$. In the case of a special pure state $\rho_{\max }=\frac{1}{2}\left[\frac{1}{\sqrt{3}}\left(\sigma_{x}+\sigma_{y}+\sigma_{z}\right)+\mathbb{I}\right]$, the equality holds.

Next, let us briefly introduce the criterion for achieving NAQC via the steering game. Suppose that Alice and Bob are two game participants and share qubits $A$ and $B$ with state $\rho_{A B}$, respectively. Alice randomly performs one of the measurements $\Pi_{i}^{b}=\left[\mathbb{I}+(-1)^{b} \sigma_{i}\right] / 2$ on qubit $A$ and obtains the outcome $b=\{0,1\}$ with the probability $p_{\Pi_{i}^{b}}=\operatorname{Tr}\left[\left(\Pi_{i}^{b} \otimes \mathbb{I}\right) \rho_{A B}\right]$. The measured state for the twoqubit state can be obtained as $\rho_{A B \mid \Pi_{i}^{b}}=\left(\Pi_{i}^{b} \otimes \mathbb{I}\right) \rho_{A B}$
$\left(\Pi_{i}^{b} \otimes \mathbb{I}\right) / p_{\Pi_{i}^{b}}$ and the conditional state for qubit $B$ is $\rho_{B \mid \Pi_{i}^{b}}=\operatorname{Tr}_{A}\left(\rho_{A B \mid \Pi_{i}^{b}}\right)$.

Alice then tells Bob to her measurement choice and outcome, and Bob's task is to measure the coherence of qubit $B$ at random in the eigenbasis of the other two of the three Pauli matrices $\sigma_{j}$ and $\sigma_{k}$. The violation in Eq. (8) implies that a single-system description of the coherence of subsystem $B$ does not exist. Thus, the criterion for achieving a NAQC of qubit $B$ can be derived via all possible probabilistic averaging methods

$N^{l_{1}}\left(\rho_{A B}\right)=\frac{1}{2} \sum_{i, j, b} p\left(\rho_{\Pi_{j \neq i}^{b}}\right) C_{l_{1}}^{\sigma_{i}}\left(\rho_{B \mid \Pi_{j \neq i}^{b}}\right)>C_{\max }$.

The NAQC quantifier is defined as

$\mathscr{N}^{l_{1}}\left(\rho_{A B}\right):=\max \left\{0, \frac{N^{l_{1}}\left(\rho_{A B}\right)-\sqrt{6}}{N_{\max }^{l_{1}}\left(\rho_{A B}\right)-\sqrt{6}}\right\}$,

upon using a convenient normalization. Herein $N_{\max }^{l_{1}}\left(\rho_{A B}\right)=$ $\max N^{l_{1}}\left(\rho_{A B}\right)$. Obviously, $\mathscr{N}^{l_{1}}\left(\rho_{A B}\right)$ ranges from 0 to 1 .

Canonically, the NAQC is treated as a stronger quantum correlation than Bell nonlocality. With regard to bipartite quantum systems, the Bell nonlocality can be detected by the famous Clauser-Horne-Shimony-Holt (CHSH) inequality [48]

$B\left(\rho_{A B}\right)=\left|\left\langle B_{\mathrm{CHSH}}\right\rangle\right|=\left|\operatorname{Tr}\left(\rho_{A B} B_{\mathrm{CHSH}}\right)\right| \leq 2$,

where $B_{\mathrm{CHSH}}$ is the Bell operator. The violation of $\mathrm{CHSH}$ inequality reflects that these states are Bell nonlocal, physical meaning of which lies in that the classical theories cannot describe the system of interest in this situation. The maximum violation of CHSH inequality [49] is $B_{\max }\left(\rho_{A B}\right)=2 \sqrt{M\left(\rho_{A B}\right)}$ with $M\left(\rho_{A B}\right)=\max _{i<j}$ $\left(u_{i}+u_{j}\right) \cdot u_{i}(i=1,2,3)$ denotes the eigenvalue of the matrix $T^{\dagger} T$ with $T^{\dagger}$ being the transposition of the correlation tensor $T$ in the Bloch representation. By a convenient normalization, the Bell nonlocality quantifier can be described as

$\mathscr{B}\left(\rho_{A B}\right):=\max \left\{0, \frac{B\left(\rho_{A B}\right)-2}{B_{\max }\left(\rho_{A B}\right)-2}\right\}$.

In Ref. [50], the criterion of quantum steering for a bipartite system is proposed as Both of Alice and Bob are with permission of measuring observables in their locations. The criterion consists of a finite sum of bilinear expectation values in a form of

$F_{n}\left(\rho_{A B}, \varsigma\right)=\frac{1}{\sqrt{n}}\left|\sum_{i=1}^{n} \operatorname{Tr}\left(\rho_{A B} A_{i} \otimes B_{i}\right)\right| \leq 1$, 


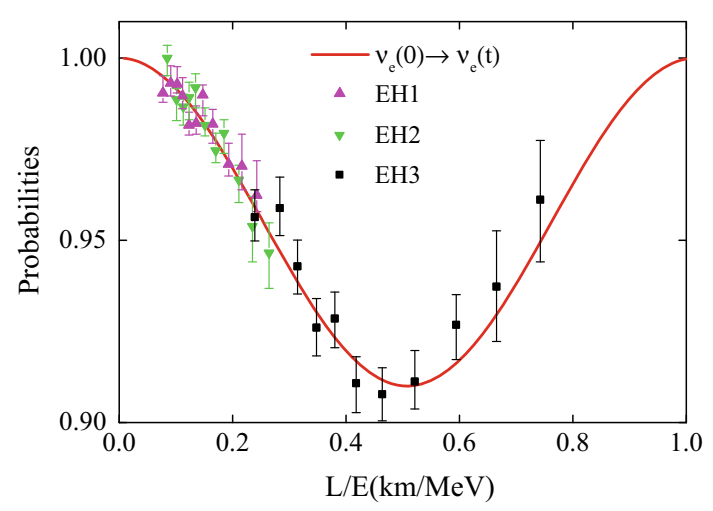

Fig. 1 The survival probability $v_{e} \rightarrow v_{e}$ (solid line, red) as a function of the distance traveled per energy $L / E$ with the initial electron flavor state. The solid line denotes the theoretical value. The data of the Daya Bay collaboration in three underground experimental halls (EH1: pink, upper triangle; EH2: green, lower triangle; EH3: black, square) are taken from Ref. [18]

where $A_{i}=\zeta_{i} \cdot \sigma$ and $B_{i}=\xi_{i} \cdot \sigma, \zeta_{i} \in \mathbb{R}^{3}$ are unit vectors, $\xi_{i} \in \mathbb{R}^{3}$ are orthonormal vectors, and $\varsigma=\left\{\zeta_{1}, \ldots, \zeta_{n}\right.$, $\left.\xi_{1}, \ldots, \xi_{n}\right\}$ denotes the set of measurement directions. A method to quantify the degree of steerability was presented by Costa and Angelo [51] based on the maximal violation of well-established steering inequality. Hereafter, we quantify the steerability of the two-qubit system in the threemeasurement scenario as follows

$\mathscr{S}_{3}\left(\rho_{A B}\right):=\max \left\{0, \frac{F_{3}\left(\rho_{A B}\right)-1}{F_{3}^{\max }\left(\rho_{A B}\right)-1}\right\}$,

where $F_{3}\left(\rho_{A B}\right)=\max _{\varsigma} F\left(\rho_{A B}, \varsigma\right)$ and $F_{3}^{\max }\left(\rho_{A B}\right)=$ $\max _{\rho_{A B}} F\left(\rho_{A B}\right)$.

3.1 NAQC, Bell nonlocality, and quantum steering in electron antineutrino oscillations

At first, we take into account preparing an electron neutrino at the initial time $t=0$, the state of the time evolution for two-flavor NOs can be expressed as

$\left|v_{e}(t)\right\rangle=a_{e e}(t)\left|v_{e}\right\rangle+a_{e \mu}(t)\left|v_{\mu}\right\rangle$.

The probabilities for finding the neutrino in the electron flavor state $v_{e}$ and the muon flavor state $v_{\mu}$ are $P_{e e}(t)=\left|a_{e e}(t)\right|^{2}$ and $P_{e \mu}(t)=\left|a_{e \mu}(t)\right|^{2}$, respectively. The effective atmospheric neutrino $\Delta m_{e e}^{2}$ measured in $v_{e}$ disappearance experiments is [52]

$\Delta m_{e e}^{2}=\sin ^{2} \theta_{12} \Delta m_{32}^{2}+\cos ^{2} \theta_{12} \Delta m_{31}^{2}$.

As can be seen from Fig. 1, the evolution of survival probabilities is plotted as a function of the ratio $L / E$, and the experimental results and the corresponding theoretical predictions are also plotted. Herein, the data is addressed from Daya Bay neutrino experiment [18]. In this scenario, EH1, EH2, and EH3 include all the measurement positions of the Daya Bay collaboration. The sites use $\beta$ decay to generate the source of electron antineutrino under conditions of different baselines and energy. The Daya Bay collaboration made use of the fully constructed Daya Bay Reactor Neutrino Experiment to measure the disappearance of electron antineutrino, and it covers energy in a range of $1-8 \mathrm{MeV}$, which implies that the range of the effective ratio $L / E$ is $[0,1]$ with the dimension $\mathrm{km} / \mathrm{MeV}$. From Fig. 1, for the short-range oscillations (with the small ratio $L / E$ ) at Daya Bay experiment, the survival probability of electron flavor state $v_{e}$ is always higher than 0.9. When the ratio at around $L / E=0.5$, the survival probability reaches the minimal value, and the oscillation probability correspondingly comes to the maximum value.

To explore the NAQC, Bell nonlocality, and quantum steering in experimentally observed electron NOs, the evolutionary state of the initial electron flavor state is expressed as

$\rho_{A B}^{e}(t)=\left(\begin{array}{llll}0 & 0 & 0 & 0 \\ 0 & \left|a_{e \mu}(t)\right|^{2} & a_{e \mu}(t) a_{e e}^{*}(t) & 0 \\ 0 & a_{e e}(t) a_{e \mu}^{*}(t) & \left|a_{e e}(t)\right|^{2} & 0 \\ 0 & 0 & 0 & 0\end{array}\right)$,

in the orthonormal basis $\{|00\rangle,|01\rangle,|10\rangle,|11\rangle\}$.

In order to obtain the quantity of the NAQC quantifier, we can resort to Eq. (9). At first, for performing Pauli measurement $\sigma_{x}$ on qubit $A$, the post-measurement states for the initial electron flavor state $\rho_{A B}^{e}(t)$ are expressed as

$\rho_{A B \mid \sigma_{x_{1}}}^{e}(t)=\left[\left(\left|x_{1}\right\rangle\left\langle x_{1}\right| \otimes \mathbb{I}\right) \rho_{A B}^{e}(t)\left(\left|x_{1}\right\rangle\left\langle x_{1}\right| \otimes \mathbb{I}\right)\right] / p_{\sigma_{x_{1}}}$,
$\rho_{A B \mid \sigma_{x_{2}}}^{e}(t)=\left[\left(\left|x_{2}\right\rangle\left\langle x_{2}\right| \otimes \mathbb{I}\right) \rho_{A B}^{e}(t)\left(\left|x_{2}\right\rangle\left\langle x_{2}\right| \otimes \mathbb{I}\right)\right] / p_{\sigma_{x_{2}}}$

with the probabilities

$p_{\sigma_{x_{1}}}=\operatorname{Tr}\left[\left(\left|x_{1}\right\rangle\left\langle x_{1}\right| \otimes \mathbb{I}\right) \rho_{A B}^{e}(t)\left(\left|x_{1}\right\rangle\left\langle x_{1}\right| \otimes \mathbb{I}\right)\right]$,

$p_{\sigma_{x_{2}}}=\operatorname{Tr}\left[\left(\left|x_{2}\right\rangle\left\langle x_{2}\right| \otimes \mathbb{I}\right) \rho_{A B}^{e}(t)\left(\left|x_{2}\right\rangle\left\langle x_{2}\right| \otimes \mathbb{I}\right)\right]$,

where $\left|i_{\kappa}\right\rangle(i=x, y, z$ and $\kappa=1,2)$ denote the eigenvectors of Pauli observable $\sigma_{i}$, respectively. The conditional state for particle $B$ can be expressed as $\rho_{B \mid \sigma_{i_{\kappa}}}=\operatorname{Tr}_{A}\left(\rho_{A B \mid \sigma_{i_{K}}}^{e}(t)\right)$. The $l_{1}$ coherence of the conditional state for $B$ in the basis of eigenvectors of Pauli observables $\sigma_{y}$ and $\sigma_{z}$ can be obtained as 
(a)

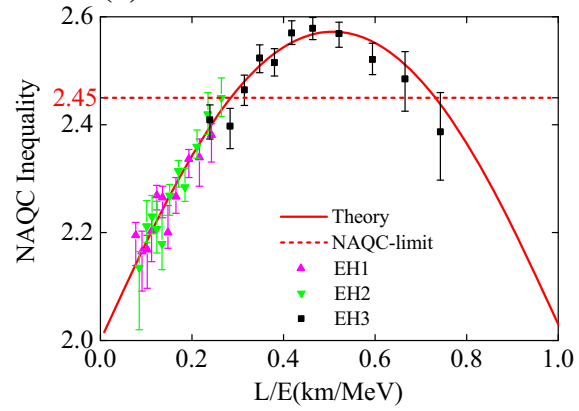

(b)

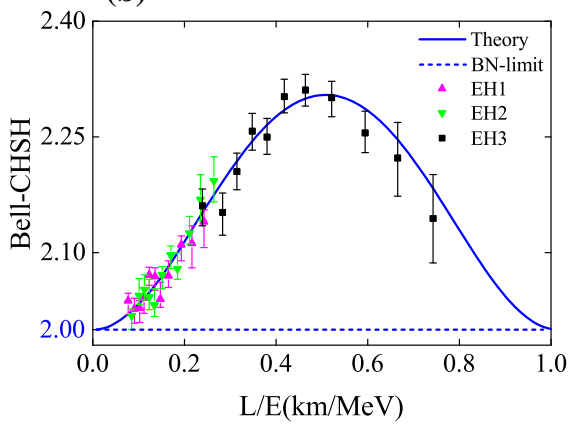

(c)

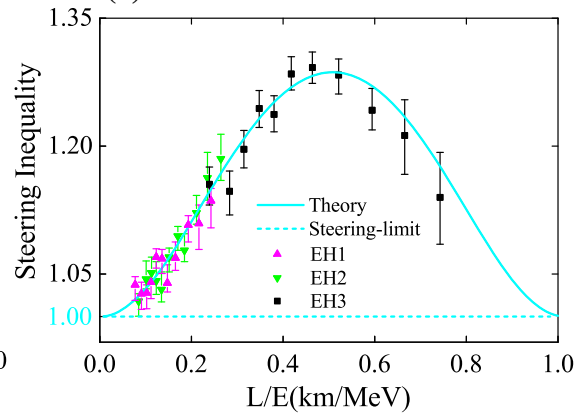

Fig. 2 NAQC inequality in Eq. (9), Bell-CHSH inequality in Eq. (11), and steering inequality in Eq. (13) in theory and experiment with error bars versus the distance traveled per energy $L / E$ for the initial electron flavor state. The red line (solid), the blue line (solid), and the azure line (solid) correspond to the left-hand sides of Eqs. (9), (11) and (13),

Likewise, the same procedure can be applied on performing Pauli measurement $\sigma_{y}$ or $\sigma_{z}$. By combining Eqs. (9) and (10), the NAQC then can be quantified by

$\mathscr{N}^{l_{1}}\left(\rho_{A B}^{e}(t)\right)=\max \left\{0, \frac{2-\sqrt{6}+2 \sqrt{\left(1-P_{e e}(t)\right) P_{e e}(t)}}{3-\sqrt{6}}\right\}$.

According to Eqs. (11), (12), (13) and (14), Bell nonlocality and quantum steering can be analytically derived as

$$
\begin{gathered}
\mathscr{B}\left(\rho_{A B}^{e}(t)\right)=\max \left\{0, \frac{\sqrt{1+4\left(\left(1-P_{e e}(t)\right) P_{e e}(t)\right)}-1}{\sqrt{2}-1}\right\}, \\
\mathscr{S}_{3}\left(\rho_{A B}^{e}(t)\right)=\max \left\{0, \frac{\sqrt{1+8\left(1-P_{e e}(t)\right) P_{e e}(t)}-1}{\sqrt{3}-1}\right\}
\end{gathered}
$$

in terms of survival probabilities.

In Fig. 2, through adopting the data from the Daya Bay neutrino experiment, we test the violations of NAQC inequality, Bell CHSH inequality, and steering inequality. The achievements of NAQC, Bell nonlocality, and quantum steering are closely related with the survival probabilities. For the ratio at around $L / E=0.5$, the violations for NAQC inequality, Bell CHSH inequality, and steering inequality maximize, meaning strong quantumness. When

respectively. The horizontal lines (dotted line, red; dotted line, blue; dotted line, azure) denote the right-hand sides of Eqs. (9), (11) and (13). Hereafter, NAQC-limit represents the violation limit with NAQC; BNlimit represents the violation limit with Bell nonlocality, and Steeringlimit represents the violation limit with quantum steering

the ratio $L / E$ in the interval $(0.2847,0.7315)$, the quantum states capture the NAQC, meanwhile Bell nonlocality and quantum steering are also captured. For the ratio $L / E$ in the regions [0, 0.2847] and [0.7315, 1], we cannot achieve the NAQC in Fig. 2a, however, Bell nonlocality and quantum steering are achieved except for $L / E=0$ and $L / E=1$ in Fig. 2b, c. It clearly shows that the quantum states capturing the NAQC must violate the $\mathrm{CHSH}$ inequality and the steering inequality, but not vice versa. This proofs that, this state must be Bell nonlocal and steerable if a quantum state is a NAQC state. Also, as to NAQC, the goodness of fit is $\chi^{2} / \mathrm{NDF}=21.4 / 30$, which correspond to $\mathrm{p}$-value equals 0.87 .

In order to better understand the relationship among NAQC, Bell nonlocality, and quantum steering in the dynamics of NO, the quantifications of them have been plotted with the ratio $L / E$ in Fig. 3. Interestingly, it directly displays that the NAQC experiences the "sudden death", neither Bell nonlocality nor quantum steering appears. From the figure, a strict hierarchical relationship among the three quantifiers is formed, namely, Bell nonlocal state is a strict subset of the steerable state and a superset of NAQC state. This manifests that the NAQC is a stronger nonclassical correlation than Bell nonlocality and quantum steering in electron neutrinos.

3.2 NAQC, Bell nonlocality, and quantum steering in muon antineutrino oscillations

If the muon neutrino is generated at the initial time $t=0$, the evolutionary state for two-flavor NOs can be expressed as

$\left|v_{\mu}(t)\right\rangle=a_{\mu e}(t)\left|v_{e}\right\rangle+a_{\mu \mu}(t)\left|v_{\mu}\right\rangle$. 


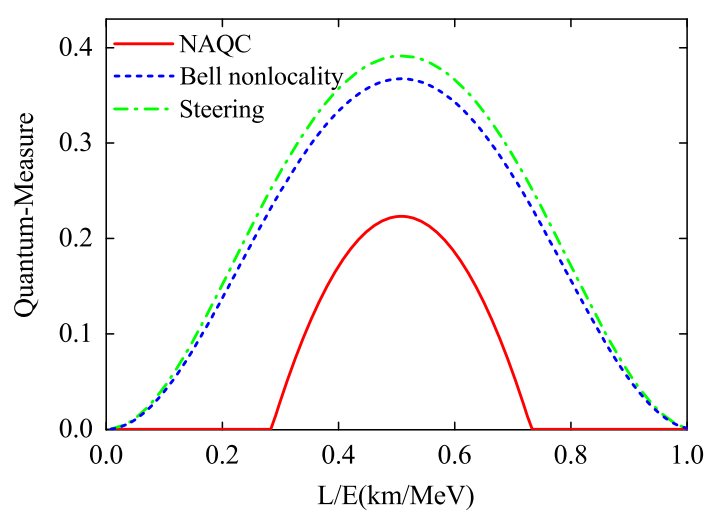

Fig. 3 The quantifiers of NAQC (solid line, red) in Eq. (10), Bell nonlocality (dashed line, blue) in Eq. (12), and Steering (dotted-dashed line, green) in Eq. (14) with respect to the distance traveled per energy $L / E$ for the initial electron flavor state

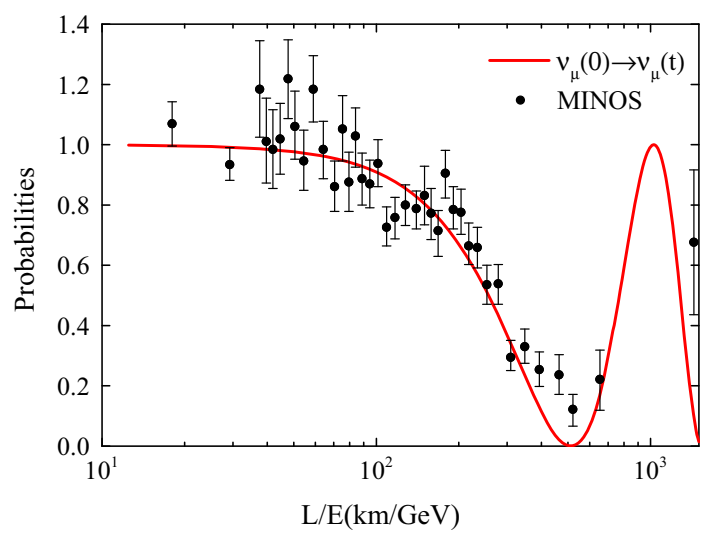

Fig. 4 The long-range survival probability $v_{\mu} \rightarrow v_{\mu}$ (solid line, red) versus the ratio $L / E$ with the initial muon flavor state. The solid line is theoretical value and the data of the MINOS Collaboration (black circle) taken from Ref. [19]

The survival probability for the muon flavor state $v_{\mu}$ is quantified by $P_{\mu \mu}(t)=\left|a_{\mu \mu}(t)\right|^{2}$ and the oscillation probability for the electron flavor state $v_{e}$ is $P_{\mu e}(t)=\left|a_{\mu e}(t)\right|^{2}$, respectively. The effective atmospheric neutrino $\Delta m_{\mu \mu}^{2}$ measured in $v_{\mu}$ disappearance experiments is [52]

$$
\begin{aligned}
\Delta m_{\mu \mu}^{2} & =\sin ^{2} \theta_{12} \Delta m_{31}^{2} \cos ^{2} \theta_{12} \Delta m_{32}^{2}+ \\
& +\sin \theta_{13} \cos \Delta \sin 2 \theta_{12} \tan \theta_{23} \Delta m_{21}^{2} .
\end{aligned}
$$

In the MINOS collaboration, the production of muon (anti)neutrino originates from the proton beams of accelerators, and the baseline occupies a fixed length of $735 \mathrm{~km}$ and the energy ranges from 0.5 to $50 \mathrm{GeV}$. It indicates that the ratio $L / E$ ranges from 15 to 1500 . The evolution of survival probabilities of the muon NO has been shown with respect to the ratio $L / E$ in Fig. 4, and the experimental results from the data of the MINOS collaboration [19] and the corresponding theoretical predictions are provided as well.
Here we investigate the NAQC, Bell nonlocality, and quantum steering in experimentally observed muon NOs. The evolutionary state for two-flavor neutrino can be rewritten with the density matrix

$\rho_{A B}^{\mu}(t)=\left(\begin{array}{llll}0 & 0 & 0 & 0 \\ 0 & \left|a_{\mu e}(t)\right|^{2} & a_{\mu e}(t) a_{\mu \mu}^{*}(t) & 0 \\ 0 & a_{\mu \mu}(t) a_{\mu e}^{*}(t) & \left|a_{\mu \mu}(t)\right|^{2} & 0 \\ 0 & 0 & 0 & 0\end{array}\right)$.

in the orthonormal basis $\{|00\rangle,|01\rangle,|10\rangle,|11\rangle\}$. Consequently, we can analytically work out the quantifications of NAQC, Bell nonlocality and quantum steering associated with survival probabilities, given by

$$
\begin{aligned}
& \mathscr{N}^{l_{1}}\left(\rho_{A B}^{\mu}(t)\right)=\max \left\{0, \frac{2-\sqrt{6}+2 \sqrt{\left(1-P_{\mu \mu}(t)\right) P_{\mu \mu}(t)}}{3-\sqrt{6}}\right\}, \\
& \mathscr{B}\left(\rho_{A B}^{\mu}(t)\right)=\max \left\{0, \frac{\sqrt{1+4\left(\left(1-P_{\mu \mu}(t)\right) P_{\mu \mu}(t)\right)}-1}{\sqrt{2}-1}\right\}, \\
& \mathscr{S}_{3}\left(\rho_{A B}^{\mu}(t)\right)=\max \left\{0, \frac{\sqrt{1+8\left(1-P_{\mu \mu}(t)\right) P_{\mu \mu}(t)}-1}{\sqrt{3}-1}\right\},
\end{aligned}
$$

respectively.

In Fig. 5, we draw the violations of NAQC inequality, Bell CHSH inequality and steering inequality as a function of the ratio $L / E$ in both theory and experiment. It is worth noting that these lines for the violations of the three inequalities are oscillating periodically and have the same periods. By theoretical analysis, we obtain that the violations of them reach maximum at the points that the survival probabilities are equal to the oscillation probabilities. This means that more quantum resources can be exploited for the propagation of neutrinos in the MINOS experiment. When the survival probabilities are zero-valued, the violations will come to minimum.

For the MINOS experiment, our theoretical curve of the survival probability is based on the standard model, without considering the effect of detectors, which plays an important role in obtaining the exmerimental data. This thereby will lead to a very small p-value. Notably, the NAQC cannot be achieved in some region of time evolution as shown in Fig. 5a, i.e., $N^{l_{1}}\left(\rho_{A B}^{\mu}(t)\right) \leq 2.45$. Whereas we can achieve Bell nonlocality and quantum steering with $L / E \neq n \times 513.215$ ( $n$ is a positive integer) as indicated in Fig. 5b, c, that is, $B\left(\rho_{A B}^{\mu}(t)\right)>2$ and $F_{n}\left(\rho_{A B}^{\mu}(t)\right)>1$ hold apart from those with several special ratios $L / E=n \times 513.215$. From viewpoint of quantum resource theory, this is because the 
(a)

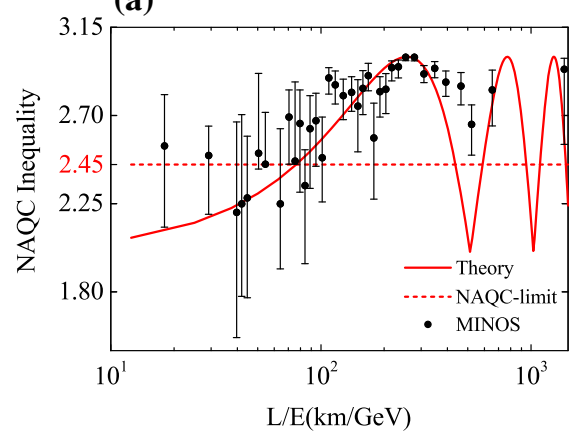

(b)

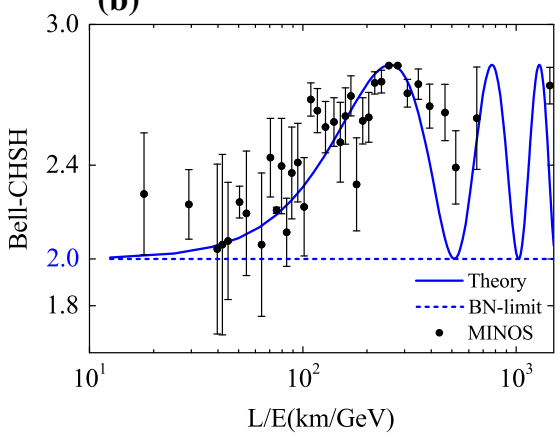

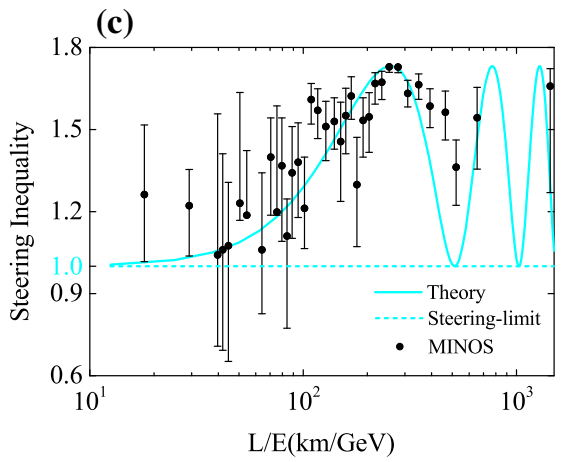

Fig. 5 NAQC inequality in Eq. (9), Bell-CHSH inequality in Eq. (11) and steering inequality in Eq. (13) in theory and experiment with error bars in MINOS collaboration (black, circle ) as a function of the ratio $L / E$ for the initial muon flavor state. The red line (solid), the blue line (solid) and the azure line (solid) represent the left-hand sides of Eqs. (9), (11) and (13), respectively. The horizontal lines (dotted line, red; dotted line, blue; dotted line, azure) are the right-hand sides of Eqs. (9), (11) and (13), respectively

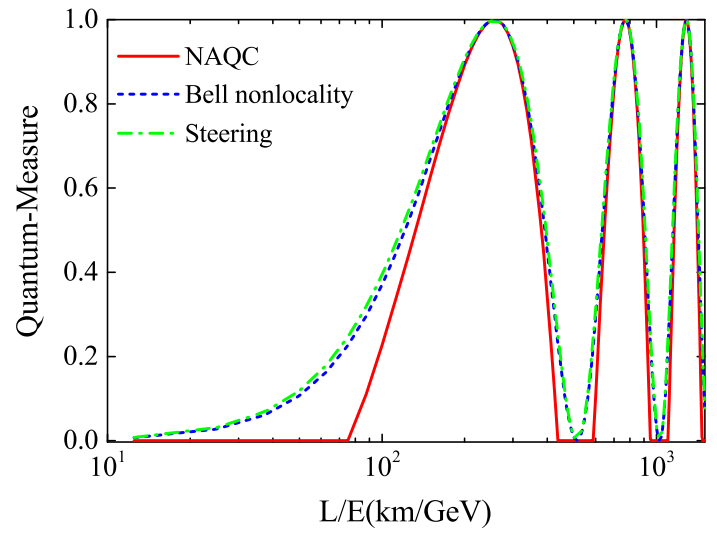

Fig. 6 The quantifiers of NAQC (solid line, red) in Eq. (10), Bell nonlocality (dashed line, blue) in Eq. (12) and Steering (dotted-dashed line, green) in Eq. (14) with respect to the distance traveled per energy $L / E$ for the initial muon flavor state

quantum correlation will minimize, when the survival probability $P_{\mu \mu}=0$ with $L / E=n \times 513.215$ yielding a product state $|01\rangle$. It reveals that a neutrino flavor state capturing the NAQC must have Bell nonlocality and quantum steering, and the criterion of the NAQC can also be considered as the indicators of Bell nonlocality and quantum steering. With this in mind, the NAQC is expected to become an important and useful physical resource in quantum information processing using neutrinos.

Further, we quantify the NAQC, Bell nonlocality and quantum steering as a function of the ratio $L / E$, shown in Fig. 6. It is obvious that the region of NAQC is the smallest in the hierarchical relationship among them. Specifically, the NAQC can vanish in some time periods, while Bell nonlocality and quantum steering not. Thus, compared with electron NOs, we also conclude that the NAQC is also a stronger quantum correlation than Bell nonlocality and quantum steering with regard to muon NOs.

\section{Conclusions}

In this paper, we have investigated the quantification of quantumness in NOs by NAQC, Bell nonlocality and quantum steering with an initial electron or muon neutrino. We analysed and compared them in experimentally observed NOs from different antineutrino sources, including Daya Bay and MINOS collaborations. The capture of NAQC are proved by experimental methods in both neutrino experiments. Moreover, we obtain that a neutrino flavor state capturing the NAQC must be Bell nonlocal and steerable, meaning that it can also be treated as the indicators of the latter two. That is, the NAQC is in essence a stronger quantum correlation than both of them. Thus, the NAQC can be regarded as reliable tools for the quantification of quantumness in the two-flavor NO over a macroscopic distance of thousands of kilometers, and we believe that it might be potentially applicable to prospective quantum information processing and telecommunications based on NOs. The strongly genuine quantum features of NOs pave the way for the further quantum information processing with neutrinos.

Most of the previous works were proposed to manipulate neutrinos for the purpose of communications, such as galactic neutrino communication [53] and submarine neutrino communication [54]. Recently, Stancil et al. [55] reported on the performance of a low-rate communications link established using the NuMI beam line and the MINERvA detector at Fermilab, illustrating the feasibility of using neutrino beams to provide a low-rate communications link. For future applications of neutrinos in quantum information, the impact of matter effect should be considered. When neutrinos propagate through a large distance of high density of matter, it feels the electron potential and the survival probability are modified. The effect is stronger for neutrinos of higher energies. For $\mathrm{MeV}$ scale solar neutrinos and a trip going through the Earth, the modification can be as large as $10 \%$ of $P_{e e}$. 
Acknowledgements This work was supported by the National Natural Science Foundation of China (Grant Nos. 61601002 and 11575001), Anhui Provincial Natural Science Foundation (Grant No. 1508085QF139), and the fund from CAS Key Laboratory of Quantum Information (Grant No. KQI201701).

Data Availability Statement This manuscript has no associated data or the data will not be deposited. [Authors' comment: This manuscript does not have associated data in a data repository.]

Open Access This article is licensed under a Creative Commons Attribution 4.0 International License, which permits use, sharing, adaptation, distribution and reproduction in any medium or format, as long as you give appropriate credit to the original author(s) and the source, provide a link to the Creative Commons licence, and indicate if changes were made. The images or other third party material in this article are included in the article's Creative Commons licence, unless indicated otherwise in a credit line to the material. If material is not included in the article's Creative Commons licence and your intended use is not permitted by statutory regulation or exceeds the permitted use, you will need to obtain permission directly from the copyright holder. To view a copy of this licence, visit http://creativecomm ons.org/licenses/by/4.0/.

Funded by SCOAP 3 .

\section{References}

1. B. Pontecorvo, Inverse beta processes and nonconservation of lepton charge. Sov. Phys. JETP 7, 172 (1958)

2. B. Pontecorvo, Inverse beta processes and nonconservation of lepton charge. Zh. Eksp. Teor. Fiz. 34, 247 (1957)

3. Z. Maki, M. Nakagawa, S. Sakata, Remarks on the unified model of elementary particles. Prog. Theor. Phys. 28, 870 (1962)

4. R. Davis Jr., D.S. Harmer, K.C. Hoffman, Search for neutrinos from the sun. Phys. Rev. Lett. 20, 1205 (1968)

5. Y. Fukuda et al. (Super-Kamiokande Collaboration), Evidence for oscillation of atmospheric neutrinos. Phys. Rev. Lett. 81, 1562 (1998)

6. M. Agostini, K. Altenmuller et al. (BOREXINO Collaboration), Comprehensive measurement of pp-chain solar neutrinos. Nature 562, 505 (2018)

7. K. Abe et al. (Super-Kamiokande Collaboration), Solar neutrino measurements in Super-Kamiokande-IV. Phys. Rev. D 94, 052010 (2016)

8. B. Aharmim et al. (SNO Collaboration), Combined analysis of all three phases of solar neutrino data from the sudbury neutrino observatory. Phys. Rev. C 88, 025501 (2013)

9. M.G. Aartsen et al. (IceCube Collaboration), Determining neutrino oscillation parameters from atmospheric muon neutrino disappearance with 3 years of IceCube DeepCore data. Phys. Rev. D 91, 072004 (2015)

10. K. Abe et al. (Super-Kamiokande Collaboration), Atmospheric neutrino oscillation analysis with external constraints in SuperKamiokande I-IV. Phys. Rev. D 97, 072001 (2018)

11. T. Araki et al. (KamLAND Collaboration), Measurement of neutrino oscillation with KamLAND: evidence of spectral distortion. Phys. Rev. Lett. 94, 081801 (2005)

12. F.P. An et al. (Daya Bay Collaboration), Observation of electronantineutrino disappearance at daya bay. Phys. Rev. Lett. 108, 171803 (2012)

13. P. Adamson et al. (MINOS Collaboration), Measurement of neutrino oscillations with the MINOS detectors in the NuMI Beam. Phys. Rev. Lett. 101, 131802 (2008)
14. P. Adamson et al. (MINOS Collaboration), Improved search for muon-neutrino to electron-neutrino oscillations in MINOS. Phys. Rev. Lett. 107, 181802 (2011)

15. K. Abe et al. (T2K Collaboration), Indication of electron neutrino appearance from an accelerator-produced off-axis muon neutrino beam. Phys. Rev. Lett. 107, 041801 (2011)

16. F. Feruglio, Pieces of the flavour puzzle. Eur. Phys. J. C 75, 373 (2015)

17. D.G. Michael et al. (MINOS Collaboration), Observation of muon neutrino disappearance with the MINOS detectors and the NuMI neutrino beam. Phys. Rev. Lett. 97, 191801 (2006)

18. F.P. An et al. (Daya Bay Collaboration), New measurement of antineutrino oscillation with the full detector configuration at daya bay. Phys. Rev. Lett. 115, 111802 (2015)

19. A.B. Sousa (MINOS and MINOS+ Collaborations), First MINOS+ data and new results from MINOS. AIP Conf. Proc. 1666, 110004 (2015)

20. L. Camilleri, E. Lisi, J.F. Wilkerson, Neutrino masses and mixings: status and prospects. Annu. Rev. Nucl. Part. Sci. 58, 343 (2008)

21. H. Duan, G.M. Fuller, Y.-Z. Qian, Collective neutrino oscillations. Annu. Rev. Nucl. Part. Sci. 60, 569 (2010)

22. M. Bustamante, J.F. Beacom, W. Winter, Theoretically palatable flavor combinations of astrophysical neutrinos. Phys. Rev. Lett. 115, 161302 (2015)

23. M. Blasone, F. Dell'Anno, S. De Siena, M. Di Mauro, F. Illuminati, Multipartite entangled states in particle mixing. Phys. Rev. D 77, 096002 (2008)

24. M.C. Gonzalez-Garcia, M. Maltoni, T. Schwetz, Updated fit to three neutrino mixing: status of leptonic CP violation. JHEP 1411, 052 (2014)

25. S. Banerjee, A.K. Alok, R. Srikanth, B.C. Hiesmayr, A quantuminformation theoretic analysis of three-flavor neutrino oscillations. Eur. Phys. J. C 75, 487 (2015)

26. A.K. Alok, S. Banerjee, S.U. Sankar, Quantum correlations in terms of neutrino oscillation probabilities. Nucl. Phys. B 909, 65 (2016)

27. D. Gangopadhyay, D. Home, A.S. Roy, Probing the Leggett-Garg inequality for oscillating neutral kaons and neutrinos. Phys. Rev. A 88, 022115 (2013)

28. J.A. Formaggio, D.I. Kaiser, M.M. Murskyj, T.E. Weiss, Violation of the Leggett-Garg inequality in neutrino oscillations. Phys. Rev. Lett. 117, 050402 (2016)

29. D. Gangopadhyay, A.S. Roy, Three-flavoured neutrino oscillations and the Leggett-Garg inequality. Eur. Phys. J. C 77, 260 (2017)

30. Q. Fu, X. Chen, Testing violation of the Leggett-Garg-type inequality in neutrino oscillations of the Daya Bay experiment. Eur. Phys. J. C 77, 775 (2017)

31. C. Budroni, C. Emary, Temporal quantum correlations and Leggett-Garg inequalities in multilevel systems. Phys. Rev. Lett. 113, 050401 (2014)

32. S. Ghosh, G. Kar, A. De Sen, U. Sen, Mixedness in the Bell violation versus entanglement of formation. Phys. Rev. A 64, 044301 (2001)

33. W.J. Munro, K. Nemoto, A.G. White, The Bell Inequality: a measure of entanglement? J. Mod. Opt. 48, 1239 (2001)

34. C. Emary, C.W.J. Beenakker, Relation between entanglement measures and Bell inequalities for three qubits. Phys. Rev. A 69, 032317 (2004)

35. R. Horodecki, P. Horodecki, M. Horodecki, K. Horodecki, Quantum entanglement. Rev. Mod. Phys. 81, 865 (2009)

36. S. Ghose, N. Sinclair, S. Debnath, P. Rungta, R. Stock, Tripartite entanglement versus tripartite nonlocality in three-qubit Greenberger-Horne-Zeilinger-Class states. Phys. Rev. Lett. 102, 250404 (2009)

37. D. Mondal, T. Pramanik, A.K. Pati, Nonlocal advantage of quantum coherence. Phys. Rev. A 95, 010301(R) (2017) 
38. Z.Y. Ding, H. Yang, H. Yuan, D. Wang, J. Yang, L. Ye, Experimental investigation of the nonlocal advantage of quantum coherence. Phys. Rev. A 100, 022308 (2019)

39. M. Richter, B. Dziewit, J. Dajka, Leggett-Garg $K_{3}$ quantity discriminates between Dirac and Majorana neutrinos. Phys. Rev. D 96, 076008 (2017)

40. J. Naikoo, A.K. Alok, S. Banerjee, S. Uma Sankar, G. Guarnieri, C. Schultze, B.C. Hiesmayr, A quantum information theoretic quantity sensitive to the neutrino mass-hierarchy. Nucl. Phys. B 951, 114872 (2020)

41. M. Blasone, F. Dell'Anno, S. De Siena, F. Illuminati, Entanglement in neutrino oscillations. Europhys. Lett. 85, 50002 (2009)

42. M. Blasone, F. Dell'Anno, S. De Siena, F. Illuminati, A fieldtheoretical approach to entanglement in neutrino mixing and oscillations. Europhys. Lett. 106, 30002 (2014)

43. X.K. Song, Y.Q. Huang, J.J. Ling, M.H. Yung, Quantifying quantum coherence in experimentally observed neutrino oscillations. Phys. Rev. A 98, 050302(R) (2018)

44. E. Nogueira, G. de Souza, A. Varizi, M. Sampaio, Quantum estimation in neutrino oscillations. Int. J. Quantun Inf. 15, 1750045 (2017)

45. C. Giunti, C.W. Kim, Fundamentals of neutrino physics and astrophysics (Oxford University Press, Oxford, 2007)

46. P. Mehta, Topological phase in two flavor neutrino oscillations. Phys. Rev. D 79, 096013 (2009)

47. T. Baumgratz, M. Cramer, M.B. Plenio, Quantifying coherence. Phys. Rev. Lett. 113, 140401 (2014)
48. J.F. Clauser, M.A. Horne, A. Shimony, R.A. Holt, Proposed experiment to test local hidden-variable theories. Phys. Rev. Lett. 23, 880 (1969)

49. R. Horodecki, P. Horodecki, M. Horodecki, Violating Bell inequality by mixed spin- $\frac{1}{2}$ states: necessary and sufficient condition. Phys. Lett. A 200, 340 (1995)

50. E.G. Cavalcanti, S.J. Jones, H.M. Wiseman, M.D. Reid, Experimental criteria for steering and the Einstein-Podolsky-Rosen paradox. Phys. Rev. A 80, 032112 (2009)

51. A.C.S. Costa, R.M. Angelo, Quantification of Einstein-PodolskyRosen steering for two-qubit states. Phys. Rev. A 93, 020103(R) (2016)

52. H. Nunokawa, S. Parke, R.Z. Funchal, Another possible way to determine the neutrino mass hierarchy. Phys. Rev. D 72, 013009 (2005)

53. J.G. Learned, S. Pakvasa, A. Zee, Galactic neutrino communication. Phys. Lett. B 671, 15 (2009)

54. P. Huber, Submarine neutrino communication. Phys. Lett. B 692, $268(2010)$

55. D.D. Stancil, P. Adamson, M. Alania et al., Demenstration of communication using neutrinos. Mod. Phys. Lett. A 27, 1250077 (2012) 